Cite this: Phys. Chem. Chem. Phys. 2015, 17, 14138

Received 14th February 2015, Accepted 24th April 2015

DOI: $10.1039 / c 5 c p 00959 f$

www.rsc.org/pccp

\section{Time-resolved IR spectroscopy of a trinuclear palladium complex in solution $\dagger$}

\author{
M. Zimmer, ${ }^{a}$ F. Rupp, ${ }^{b}$ P. Singer, ${ }^{b}$ F. Walz, ${ }^{c}$ F. Breher, ${ }^{\star c}$ W. Klopper, ${ }^{\star d}$ R. Diller ${ }^{\star b}$ \\ and M. Gerhards*a
}

\begin{abstract}
This paper presents a combined spectroscopic and theoretical analysis of a trinuclear $\left[\mathrm{Pd}_{3}\left\{\mathrm{Si}\left(\mathrm{mt}^{\mathrm{Me}}\right)_{3}\right\}_{2}\right]$ complex ( $\mathrm{mt}^{\mathrm{Me}}=$ methimazole) which has been demonstrated to be a potential catalyst for coupling reactions. It is a highly symmetric model system $\left(D_{3}\right.$ in the electronic ground state) for the investigation of electronic states and the structure of polynuclear transition metal complexes. Different time-resolved IR spectroscopic methods covering the femtosecond up to the microsecond range as well as density functional computations are performed to unravel the structure and character of this complex in the electronically excited state. These are the first time-resolved IR studies on a trinuclear Pd complex. Based on the interplay between the computational results and those from the IR studies a ${ }^{3} A$ state is identified as the lowest lying triplet state which has $C_{2}$ symmetry.
\end{abstract}

\section{Introduction}

Polynuclear transition metal complexes are known to have chemically interesting properties and applications, e.g. in catalysis ${ }^{1-4}$ or light harvesting processes, ${ }^{5,6}$ which can be explored in photochemical and photophysical investigations. ${ }^{7-9}$ The afore-mentioned properties can be influenced by cooperative effects between the metal centers, ${ }^{10-13}$ i.e. the interactions (couplings) between the metal centers and the ligand(s) have to be investigated. These investigations require versatile spectroscopic techniques and detailed theoretical analyses. In recent years structure-sensitive $\mathrm{X}_{\text {-ray }}{ }^{14-18}$ and vibrational spectroscopy ${ }^{19-28}$ with sub-ps up to $\mu$ s time resolution have been applied to explore structure and dynamics of electronically excited transition metal containing complexes.

In this context a very interesting trinuclear palladium complex ${ }^{29}$ (1, Fig. 1) has been investigated by our research groups with a variety of state-of-the-art photophysical and computational methods. $^{30}$ These analyses gave a detailed insight into the

\footnotetext{
${ }^{a}$ Chemistry Department and Research Center Optimas, TU Kaiserslautern, Erwin-Schrödinger-Straße 52, 67663 Kaiserslautern, Germany.

E-mail: gerhards@chemie.uni-kl.de; Fax:+49631 2052750; Tel: +496312052537

${ }^{b}$ Physics Department, TU Kaiserslautern, Erwin-Schrödinger-Straße 46, 67663

Kaiserslautern, Germany. E-mail: diller@physik.uni-kl.de; Fax: +49 631 2053902; Tel: +496312052323

${ }^{c}$ Institute of Inorganic Chemistry, Karlsruhe Institute of Technology (KIT),

Engesserstr. 15, 76131 Karlsruhe, Germany.E-mail: breher@kit.edu;

Fax: +49721 60847021; Tel: +4972160844855

${ }^{d}$ Institute of Physical Chemistry, Karlsruhe Institute of Technology (KIT), Fritz-Haber-Weg 2, 76131 Karlsruhe, Germany.E-mail: klopper@kit.edu; Fax: +49721 60847225; Tel: +4972160847263

$\dagger$ Electronic supplementary information (ESI) available. See DOI: 10.1039/c5cp00959f
}

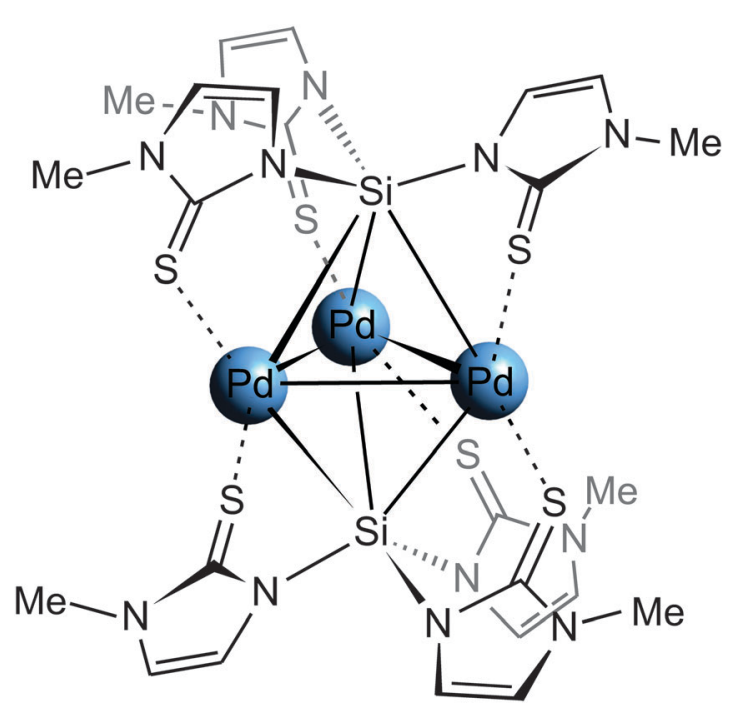

Fig. 1 Structural arrangement of $\left[\mathrm{Pd}_{3}\left\{\mathrm{Si}\left(\mathrm{mt}^{\mathrm{Me}}\right)_{3}\right\}_{2}\right]$ (1).

photophysical properties and into the electronically excited states. Based on these investigations we could characterize the lowest lying triplet state from which a long-lived $\left(\tau_{\mathrm{Ar}}=1.3-1.7 \mu \mathrm{s}\right.$, $\left.\tau_{\text {Air }}=222 \pm 3 \mathrm{~ns}\right)^{30}$ luminescence occurs. Extensive theoretical work was involved to obtain conclusions on the electronically excited states of $\mathbf{1}$. However, no conclusive investigations on the structures of the electronic states have been performed up to now, which would yield more precise information on these states and thus a better and more reliable assignment. For the investigations presented here we use structure sensitive timeresolved IR spectroscopy from the femto- to the microsecond 
time range in combination with further theoretical analyses that go beyond our previous work; ${ }^{30}$ this is the first application of such a strategy to a trinuclear Pd complex. Our chosen combination of methods is especially suited to analyse the structure of the complex in its ground and excited states.

To cover the nano- and microsecond range, time-resolved step-scan FTIR spectroscopy (TR-FTIR) is applied. Publications of TR-FTIR measurements on transition metal complexes exist since the early 1990s. ${ }^{22-25}$ Most of those investigations are about electronically excited states, especially with respect to metal-toligand charge transfer (MLCT) transitions in complexes including one or two metal centers. In these early investigations so called "reporter" ligands (carbonyl and cyanide) have been introduced which bear the advantage of a huge oscillator strength for the $\nu(\mathrm{CO})$ and $\nu(\mathrm{CN})$ stretching vibration(s) and a high sensitivity for structural changes. ${ }^{22}$ Investigated systems without a "reporter" ligand are for example $\mathrm{Ru}(\mathrm{bpy})$ complexes (bpy $=2,2$ '-bipyridine) including the well analyzed $\left[\mathrm{Ru}(\mathrm{bpy})_{3}\right]^{2+26,27}$ Regarding the number of metal atoms very few examples for tri- and tetrametallic investigations with TR-FTIR can be found in the literature, some of them focus on the energy transfer in rhenium complexes, e.g. $\left[(\mathrm{bpy})(\mathrm{CO})_{3} \operatorname{Re}\left(\right.\right.$ trans $\left.^{-d p p e n e}\right) \operatorname{Re}(\mathrm{phen})(\mathrm{CO})_{2}\left(\right.$ trans $^{-}$ dppene $) \operatorname{Re}($ bpy $\left.)(\mathrm{CO})_{3}\right]^{3+}$ (phen $=$ phenanthroline, dppene $=1,2-$ bis(diphenylphosphino)ethene) and $\mathrm{Re}_{4}(\mathrm{CO})_{12}\left(4,4^{\prime}-\mathrm{bpy}\right)_{4} \mathrm{Cl}_{4} \cdot{ }^{24,28}$

Having none of the above mentioned "reporter" groups in our system (1) our investigations are even more demanding. The transient (step-scan) FTIR spectroscopy still benefits from the advantages of the FTIR technique among them the possibility to record a complete spectral region simultaneously (multiplex advantage). Thus it is possible to capture the whole mid-IR region (and in principle also NIR and FIR). In our case the mid-IR region, especially from 1600 to $1100 \mathrm{~cm}^{-1}$, is of interest.

After a detector rise time of about 30 ns our step-scan set up enables us to perform measurements with a resolution of about 10 ns (see Section 2). Beyond this, ultrafast transient IR spectroscopy is applied to the trinuclear palladium complex to investigate the primary photoinduced vibrational dynamics in the femto and picosecond time domain, thereby covering the entire excited state lifetime from its very beginning. Femtosecond transient IR spectroscopy has been applied successfully to the investigation of divers photoinduced phenomena in metal complexes such as CO release,$^{31-33}$ excited state dynamics in $\mathrm{Cr}(\mathrm{acac})_{3}{ }^{34}$ and a $\mathrm{Ru}(\mathrm{II})$ catalyst $^{35}$ as well as high-spin state formation in $\mathrm{Fe}(\mathrm{II})$ spin-crossover systems. ${ }^{21}$ Thereby ultrafast vibrational dynamics can be related to electronic dynamics as intersystem crossing and internal conversion. ${ }^{36,37}$

\section{Experimental methods}

The $\mathrm{Pd}_{3}$ complex (1) was prepared following the synthesis described in ref. 29. DMSO-d 6 ( $<0.02 \%$ water, $99.8 \% \mathrm{D})$ was purchased from VWR Chemicals and purged with argon prior to usage. All experiments described in Sections 2.1 and 2.2 were performed at room temperature.

\subsection{Time-resolved step-scan FTIR spectroscopy}

For the time-resolved FTIR experiments a Bruker VERTEX 80v spectrometer equipped with a step-scan interferometer module was used (for a detailed description of the experimental set-up, $c f$. Fig. S1 of the ESI $\dagger$ ). The spectrometer, using a liquid-nitrogen cooled mercury-cadmium-telluride (MCT) detector connected to a fast preamplifier, was coupled with a Q-switched Nd:YAG laser (Lumonics HY750, frequency tripled to $355 \mathrm{~nm}$ ) that generates excitation pulses with a half-width of about $10 \mathrm{~ns}$ and a repetition rate of $10 \mathrm{~Hz}$. The timing of the laser pulse and the step-scan triggering was performed by a Stanford Research Systems DG535 delay generator. The UV pump beam was adjusted to have an overlap with the spectrometer's IR probe beam inside the flow cell. To avoid backscattering of the UV pulse into the detector or interferometer compartment, AR-coated germanium filters were placed inside the sample compartment.

A mM solution of 1 in argon purged DMSO- $\mathrm{d}_{6}$ was pumped by a peristaltic pump (Masterflex Easy-Load II, variable flow rate) through an optical cell that is part of a closed-cycle continuous flow tubing system including a sample reservoir. The cell was composed of housing, $\mathrm{CaF}_{2}$ windows, separated by two Mylar ${ }^{\mathbb{R}}$ spacers with a variable thickness (0.006 to $0.5 \mathrm{~mm})$. In our case $0.26 \mathrm{~mm}$ spacers and a flow rate of about $40 \mathrm{~mL} \mathrm{m^{-1 }}$ are chosen. The sample cell as well as the sample reservoir were purged by argon to assure an excited state lifetime of $\tau_{\mathrm{Ar}}=$ 1.3-1.7 $\mu \mathrm{s}$, since it is quenched by oxygen to $\tau_{\text {Air }}=222 \mathrm{~ns}^{30}{ }^{30}$ The $355 \mathrm{~nm}$ excitation laser beam was attenuated to $0.6-0.8 \mathrm{~mJ}$ per shot at a diameter of about $9 \mathrm{~mm}$. The temporal resolution was set to $20 \mathrm{~ns}$ for these measurements. Five adjacent $20 \mathrm{~ns}$ intervals were joined and averaged, so a temporal resolution of $100 \mathrm{~ns}$ was obtained. The step-scan experiment was triggered 400 ns before the Q-Switch of the laser was triggered which means that the laser beam reached the sample $600 \mathrm{~ns}$ after the experiment starts. Hence this time was set as zero point in all spectra. A total number of 465 coadditions at each interferogram point was recorded. The spectral region was limited by undersampling to $0-2633 \mathrm{~cm}^{-1}$ with a spectral resolution of $2 \mathrm{~cm}^{-1}$ (resulting in 2665 interferogram points). For the prevention of problems by performing a Fourier transformation an IR longpass filter (transmission 4.73-9.00 $\mu \mathrm{m}$ ) was used (i.e. no IR intensity outside the measured region should be observed).

Both amplified AC and DC signals of the MCT detector were fed to a 14-bit transient recorder board (Spectrum Germany, M3I4142, $400 \mathrm{MS} / \mathrm{s}$ ), which in turn was connected to an external computer running Bruker OPUS software for spectrometer control and data manipulation/analysis. For data analysis the recorded DC and AC spectra were used to calculate the $\Delta A$ (change in absorbance) spectra with:

$$
\Delta A=-\log \left(\frac{\mathrm{AC}+\mathrm{DC} \times \gamma}{\mathrm{DC} \times \gamma}\right)
$$

with the factor $\gamma$ that is compensating for different amplifications of AC and DC channel (for further description, see ESI $\dagger$ ). The resulting spectra show peaks with positive and negative sign. Negative bands are bleach bands resulting from the 
depletion of ground state vibration. Positive bands belong to vibrations of the excited species.

\subsection{Ultrafast transient IR absorption}

For femtosecond mid-IR experiments, the complex was dissolved at concentration equivalent to an $\mathrm{OD}=2$ at $330 \mathrm{~nm}$ in dimethylsulfoxide (DMSO) for probe around $1166 \mathrm{~cm}^{-1}$ and in deuterated DMSO (DMSO- $\mathrm{d}_{6}$ ) for probe around 1453, 1295, $1378 \mathrm{~cm}^{-1}$ (see Section 4). The solution was placed between two UV-grade $\mathrm{CaF}_{2}$ windows (Crystal $\mathrm{GmbH}$ ) of $2.54 \mathrm{~cm}$ in diameter with $250 \mu \mathrm{m}$ path length. Before and after each femtosecond transient experiment steady state UV/Vis spectra (JASCO, V-670) were recorded to ensure sample integrity.

The femtosecond pump-probe experiments were based on a Ti:sapphire regenerative amplifier system to generate ultrashort pump and probe pulses (CPA-2001, Clark MXR: rep. rate $1 \mathrm{kHz}$, pulse energy $1 \mathrm{~mJ}$, central wavelength $775 \mathrm{~nm}$, pulse duration 150 fs (FWHM)).

A home-build two stage non-collinear optical parametric amplifier was used to generate femtosecond pump pulses at $330 \mathrm{~nm}$ with energy of 250-300 nJ at the sample position. Another portion of the fundamental beam was fed in a two stage optical parametric amplifier with consecutive difference frequency generation, yielding the spectrally tunable probe pulse between 6-12 $\mu \mathrm{m}$ with a spectral width of $50-60 \mathrm{~cm}^{-1}$ as described before. ${ }^{38}$ Pump and probe pulses were superimposed in the sample with focal width of about 200 and $100 \mu \mathrm{m}$, respectively. The sample was rotated to exchange the excited volume between two consecutive laser shots. The transmitted mid-IR probe light was spectrally dispersed and detected by a polychromator with a 32-element MCT (mercury-cadmium-telluride) detector array (InfraredSystems-Development) with $\approx 2 \mathrm{~cm}^{-1}$ spectral width per element. In all experiments the pump beam was chopped at half the repetition rate $(500 \mathrm{~Hz})$, allowing the calculation of pump-induced difference absorbance spectra $\Delta \mathrm{OD}(\lambda, t)$ on a shot-to-shot basis as a function of probe wavelength and pump-probe delay. Time zero of the experiment was determined via a transient absorption experiment on a thin silicon wafer. The pump/probe cross correlation time was typically $\approx 350$ fs (FWHM, Gauss).

The transient data was analyzed by a global fit routine, describing the time dependent absorbance change $\Delta \operatorname{OD}(\lambda, t)$ as a sum of exponential decay functions

$$
\Delta \mathrm{OD}(\lambda, t)=A_{0}(\lambda)+\sum_{i=1}^{n} A_{i}(\lambda) \cdot \mathrm{e}^{-\frac{t}{\tau_{i}}}
$$

with amplitudes $A_{i}(\lambda)$ as decay associated spectra (DAS), $\tau_{i}$ as the corresponding time constants and $A_{0}(\lambda)$ as the difference spectrum after "infinite" delay time.

\section{Theory}

Quantum-chemical calculations were performed with the TURBOMOLE program package ${ }^{39}$ at the level of density-functional theory (DFT) using the hybrid functional B3LYP. The calculations were performed in exactly the same basis as used in ref. 30, that is, dhf-TZVP-2c for Pd, $\mathrm{S}$, and $\mathrm{Si}$, and def2-SV(P) for $\mathrm{H}, \mathrm{C}$, and $\mathrm{N}$. This basis is referred to as "TZVP" for short. In ref. 30, the geometries of the ${ }^{3} \mathrm{~A}_{1}$ and ${ }^{3} \mathrm{~A}_{2}$ excited states as well as the ${ }^{1} \mathrm{~A}_{1}$ ground state were optimized in the framework of self-consistent Kohn-Sham calculations $(\triangle \mathrm{SCF})$ at the B3LYP/TZVP level in $D_{3}$ symmetry. Subsequently, in the course of the present work, we computed the harmonic vibrational frequencies at these geometries using a parallelized version ${ }^{40}$ of the module AOFORCE, ${ }^{41}$ which allows for the analytical calculation of force constants. For the ${ }^{1} \mathrm{~A}_{1}$ ground state, we have found that all computed harmonic vibrational frequencies are real, meaning that the $D_{3}$ equilibrium structure is a true minimum on the potential energy hypersurface. For each of the two triplet excited states, however, we obtained one (two-fold degenerate) imaginary frequency of $e$ symmetry (26i $\mathrm{cm}^{-1}$ for the ${ }^{3} \mathrm{~A}_{1}$ state and $122 i \mathrm{~cm}^{-1}$ for ${ }^{3} \mathrm{~A}_{2}$ ). Therefore, these states were re-optimized in $C_{2}$ symmetry, now yielding true minima with real harmonic vibrational frequencies. In $C_{2}$ symmetry, the ${ }^{3} \mathrm{~A}_{1}\left(D_{3}\right)$ state becomes a ${ }^{3} \mathrm{~A}$ state, whose adiabatic $\triangle \mathrm{SCF}$ energy is found $1.79 \mathrm{eV}$ above the well depth of the ground state (this value was $1.81 \mathrm{eV}$ for its $D_{3}$-symmetric counterpart) while the ${ }^{3} \mathrm{~A}_{2}\left(D_{3}\right)$ state becomes a ${ }^{3} \mathrm{~B}$ state, whose adiabatic energy is found $2.01 \mathrm{eV}$ above the ground state (this value was $2.32 \mathrm{eV}$ in $D_{3}$ symmetry). Hence, the energy lowering due to the geometry relaxation in $C_{2}$ symmetry amounts to 0.02 and $0.31 \mathrm{eV}$ for the ${ }^{3} \mathrm{~A}$ and ${ }^{3} \mathrm{~B}$ states, respectively. In the ${ }^{3} \mathrm{~A}$ state, the three Pd atoms form an isosceles triangle with two equal sides of $270.2 \mathrm{pm}$ and one longer side of $275.4 \mathrm{pm}$ while in the ${ }^{3} \mathrm{~B}$ state, they form an isosceles triangle with two equal sides of $285.9 \mathrm{pm}$ and one shorter side of $281.3 \mathrm{pm}$ (in $D_{3}$ symmetry, the Pd-Pd distances were $271.5 \mathrm{pm}$ and $288.2 \mathrm{pm}$, respectively, in the ${ }^{3} \mathrm{~A}_{1}$ and ${ }^{3} \mathrm{~A}_{2}$ states).

For gaining insight into the observed energy lowering due to geometry relaxation, we computed a correlation diagram ( $c f$. ESI, $\dagger$ Fig. S2) of the frontier orbitals as a function of a distortion coordinate $x$, which is defined as follows: $x=0$ corresponds to an unbiased $D_{3}$-symmetric geometry that is obtained by averaging the ${ }^{3} \mathrm{~A}_{1}$ and ${ }^{3} \mathrm{~A}_{2}$ geometries optimized in ref. $30 ; x=-1$ corresponds to the $C_{2}$-symmetric geometry of the ${ }^{3} \mathrm{~B}$ state and $x=+1$ corresponds to the $C_{2}$-symmetric geometry of the ${ }^{3} \mathrm{~A}$ state. Separate linear interpolations of the structures are performed between the $x=-1$ and $x=0$ geometries on the one hand and between the $x=0$ and $x=$ +1 geometries on the other. The LUMO is stabilized for both positive $(x>0)$ and negative $(x<0)$ distortions. This can be understood as pseudo-Jahn-Teller effect ${ }^{42}$ due to interactions with a close-lying two-fold degenerate e level (LUMO+1, 72e). At the same time, the HOMO is destabilized towards $x=+1$ while the HOMO-1 is destabilized towards $x=-1$. The latter even becomes the HOMO in the ${ }^{3} \mathrm{~B}$ geometry. The interplay between destabilization of the relevant ground-state occupied orbitals and stabilization of the LUMO appears to be responsible for the energy lowering upon distortion. Consistent with the correlation diagram, Fig. S3 (ESI $\dagger$ ) shows the energies of the excited states of interest as a function of the distortion coordinate, as obtained from TDDFT computations at the B3LYP/TZVP level using the lowest triplet state as reference.

The Cartesian coordinates of the ground state and the two triplet states are given in the $\mathrm{ESI} \dagger$ (Tables S1-S3). 


\section{Spectroscopic results and discussion}

\section{Static IR spectra}

In the spectral region from 1150 to $1550 \mathrm{~cm}^{-1}$ four IR absorption bands belonging to 1 , at $1172,1301,1383,1457 \mathrm{~cm}^{-1}$ are identified (see Fig. S4, ESI $\dagger$ and lower trace of Fig. 2 in the region down to $1250 \mathrm{~cm}^{-1}$ ); furthermore a broad, not well structured absorption region from $1390-1450 \mathrm{~cm}^{-1}$ is observed. In comparison to the DFT calculations the four defined peaks can be assigned to $\delta(\mathrm{C}-\mathrm{H})_{\text {aliphatic }} 1457 \mathrm{~cm}^{-1}\left(1454 \mathrm{~cm}^{-1}\right), \nu(\mathrm{C}-\mathrm{N}) 1301 \mathrm{~cm}^{-1}$ $\left(1300 \mathrm{~cm}^{-1}\right)$ and skeletal vibrations 1383 and $1172 \mathrm{~cm}^{-1}$ (1379 and $1159 \mathrm{~cm}^{-1}$ ), calculated values in parentheses (scaling factor 0.98 , see below).

\section{Nano- and microsecond time regime}

After laser excitation five bleach bands appear at 1174, 1303, 1385, 1402 and $1458 \mathrm{~cm}^{-1}$ (Fig. 2 and 3) in the step-scan FTIR spectrum, which can definitely be assigned to the electronic ground state vibrations. (Remark: the values observed in depletion differ by a few wavenumbers from the values obtained in the static FTIR spectrum due to a significant spectral overlap of the groundand electronically excited state vibrational bands. This is illustrated in Fig. S5, ESI $\dagger$ ). In addition, four new bands appear that are $9-14 \mathrm{~cm}^{-1}$ red shifted at $1162,1289,1373$ and $1449 \mathrm{~cm}^{-1}$. These bands originate from the electronically excited species. Furthermore the relative depletion intensities of the $\Delta A$ peaks match that of the steady state spectrum (see Fig. 2 and Fig. S6, ESI†).

In the first $100 \mathrm{~ns}$ interval after excitation the bands at 1303/ $1289,1385 / 1373$ and $1458 / 1449 \mathrm{~cm}^{-1}$ are already observable (see inset of Fig. 2). To obtain a better $\mathrm{S} / \mathrm{N}$ ratio and since no dynamics in this temporal range are visible spectra are also averaged to 500 or $1000 \mathrm{~ns}$ steps. The signal disappears completely after about $3.5 \mu \mathrm{s}$, confirming the TCSPC lifetime measurements ${ }^{30}$ and giving a hint that the argon purged sample chamber and argon purged housing of the sample reservoir prevent the sample from oxygen. (For a better illustration of the decay within microseconds, the

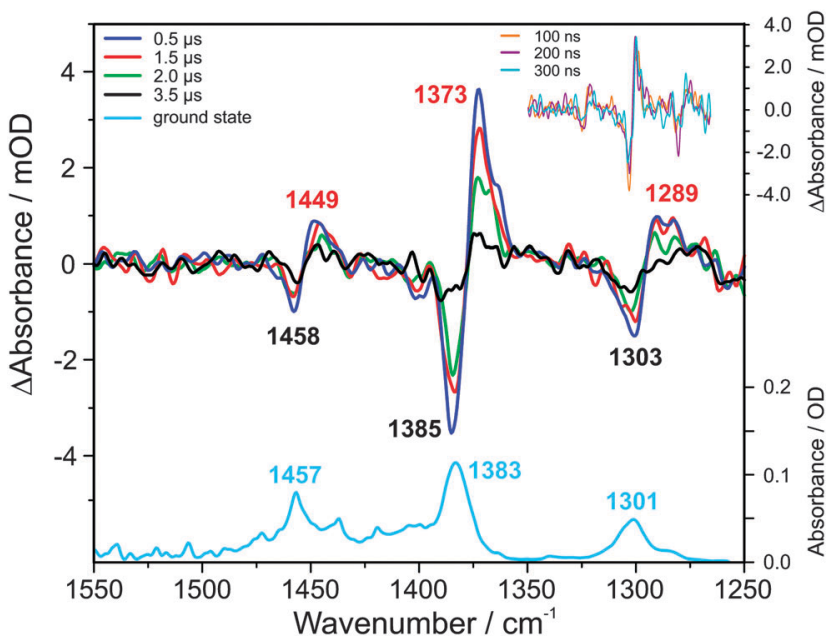

Fig. 2 Bottom: steady state FTIR, solvent DMSO- $d_{6}$ subtracted; top: TR-FTIR spectra, 0.5, 1.5, 2.0 and $3.5 \mu$ s after excitation at $355 \mathrm{~nm}$; inset: TR-FTIR spectra, $0.1,0.2$ and $0.3 \mu \mathrm{s}$ after excitation at $355 \mathrm{~nm}$.
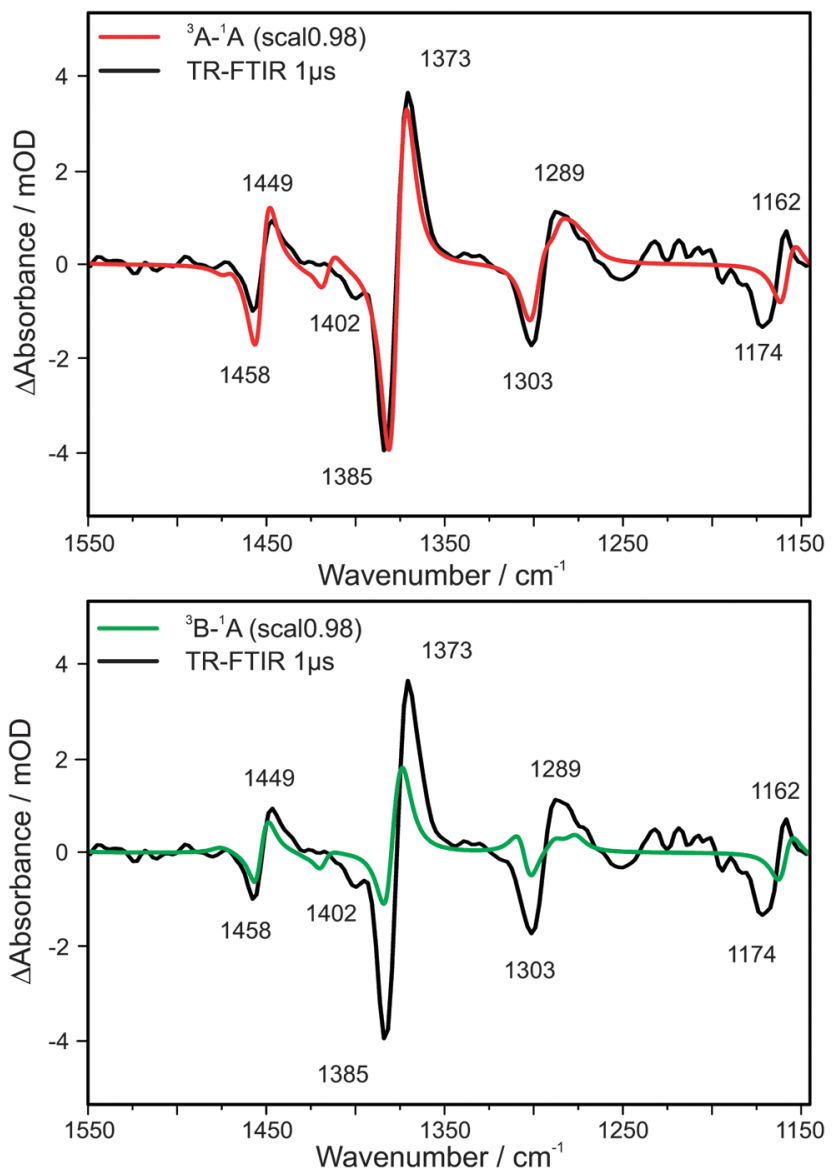

Fig. 3 TR-FTIR spectrum $1.0 \mu$ s after excitation; top: in comparison with calculated excitation into a ${ }^{3} \mathrm{~A}$ state; bottom: in comparison with calculated excitation into ${ }^{3}{ }^{3}$ state.

transient IR spectra up to $3.5 \mu \mathrm{s}$ are shown in steps of $500 \mathrm{~ns}$ in the ESI, $\dagger c f$. Fig. S7).

In order to compare calculated harmonic frequencies with experimental values the computed vibrational frequencies have to be scaled. A typical empirical factor for the chosen theoretical ansatz (cf. Section 3) is 0.98 (cf. e.g. ref. 43). The reliability of this factor can be tested by fitting the calculated IR spectrum of the electronic ground state $\left({ }^{1} \mathrm{~A}\right)$ as well as the spectra of the electronically excited states $\left({ }^{3} \mathrm{~A}\right.$ and $\left.{ }^{3} \mathrm{~B}\right)$ to the experimental values. Indeed the best agreement is achieved for a factor of 0.98 . (In case of the ${ }^{3} \mathrm{~A}$ state the scaling factors vary between 0.979 and 0.983 whereas for the ${ }^{3} \mathrm{~B}$ state the variation is larger, between 0.970 and 0.983 , indicating a better description of the experimental frequencies for the ${ }^{3} \mathrm{~A}$ state.)

In the DFT calculations a red shift of the ${ }^{3} \mathrm{~A}$ state vibrations compared to the ground state ${ }^{1} \mathrm{~A}$ (modes) in the order of about $10 \mathrm{~cm}^{-1}$ is observable, for example $16 \mathrm{~cm}^{-1}$ for the 1285/ $1301 \mathrm{~cm}^{-1}$ peaks or $7 \mathrm{~cm}^{-1}$ for the $1373 / 1380 \mathrm{~cm}^{-1}$ peaks (Fig. S8). In contrast, the vibrational modes of the ${ }^{3} \mathrm{~B}$ state are closer to the values of the ${ }^{1} \mathrm{~A}$ state. It should be mentioned that the investigated spectral region shows small but significant differences for the calculated ${ }^{3} \mathrm{~A}$ and ${ }^{3} \mathrm{~B}$ spectra. Other parts of the spectrum are either less significant or cannot be observed with our experimental set-ups ( $c f$. Fig. S9 of the ESI†). 
In order to quantify the comparison between calculated and experimentally observed frequencies the calculated spectrum of the electronic ground state $\left({ }^{1} \mathrm{~A}\right)$ has to be subtracted from the calculated spectra obtained for the ${ }^{3} \mathrm{~A}$ and ${ }^{3} \mathrm{~B}$ states. In order to simulate the vibrational spectra, Lorentzian functions are used. By fixing the band positions (scaling factor 0.98), different full widths at half maximum (FWHM) are chosen. By testing a constant width or variable widths (between 10 and $20 \mathrm{~cm}^{-1}$ ) within one fit it turns out that $14 \mathrm{~cm}^{-1}$ is the best average value. The goodness of the fit can be described by the $R^{2} / \chi^{2}$ parameter which is about a factor of three to four better for the ${ }^{3} \mathrm{~A}$ state compared to ${ }^{3} \mathrm{~B}$ (by using a scaling factor of 0.98 and a constant band width of $14 \mathrm{~cm}^{-1}$ or a variable band width between 10 and $20 \mathrm{~cm}^{-1}$ optimized for each transition). Fig. 3 shows the comparison of experiment and theory: Fig. 3 (top) ground state $\left({ }^{1} \mathrm{~A}\right)$ subtracted from ${ }^{3} \mathrm{~A}$ and Fig. 3 (bottom) ${ }^{1} \mathrm{~A}$ subtracted from ${ }^{3} \mathrm{~B}$. It can be seen that the calculated band positions (relative to the experimental values) are better predicted for the ${ }^{3} \mathrm{~A}$ state, especially in the case of the transitions at 1289, 1373 and $1449 \mathrm{~cm}^{-1}$. In case of the ${ }^{3} \mathrm{~B}$ state also an additional band around $1310 \mathrm{~cm}^{-1}$ would be expected which is not observed in the experimental spectrum. Furthermore, the calculated difference spectra reveal also that only the ${ }^{3} \mathrm{~A}$ state gives an appropriate description of the experimentally observed band intensity ratios, especially for the transitions at $1373 / 1385 \mathrm{~cm}^{-1}$. In case of this most prominent pair of transitions both increase and depletion in the $\Delta A$ spectrum are of similar intensity for the ${ }^{3} \mathrm{~A}$ state (in agreement with the experiment) whereas for the ${ }^{3} \mathrm{~B}$ state the increase should be by a factor of 3 larger than the depletion. Taking the qualitative and quantitative aspects of calculated frequencies and intensities into account, it can be concluded that the excited state probed on the nano- and microsecond time scale is $a^{3} \mathrm{~A}$ state.

Another experiment was performed with a frequency doubled Nd:YLF laser (edgewave INNOSLAB IS8 II - ET, output of about $0.4 \mathrm{~mJ}$ per shot at $261.5 \mathrm{~nm}$ ) that was operated at $100 \mathrm{~Hz}$. According to our previous experimental study ${ }^{30}$ it is known that the UV/Vis spectrum shows two strong and broad absorptions: the first electronic transition has its maximum around $333 \mathrm{~nm}$ and a second one is around $262 \mathrm{~nm}$. By exciting the second electronic transition with a wavelength of $261.5 \mathrm{~nm}$, the same IR transitions as for the excitation of the first electronic transitions (with $355 \mathrm{~nm}$ ) are observed in the step-scan FTIR spectrum. These finding clearly show that after electronic excitation the same long-lived electronic state is reached.

\section{Femto- and picosecond time regime}

In our earlier work, ${ }^{30}$ femtosecond transient absorption in the UV/Vis already showed the ultrafast formation of an electronically excited state within much less than 1 ps with no subsequent spectral changes up to $700 \mathrm{ps}$. In order to investigate the formation of this long lived state via its IR spectrum transient IR spectroscopy is performed. Fig. 4 (top) displays IR difference absorption signals at selected delay times of 0.5 and $340 \mathrm{ps}$ after photoexcitation at $330 \mathrm{~nm}$ between 1145 and $1480 \mathrm{~cm}^{-1}$. Fig. 4 (bottom) depicts two kinetic traces at 1366 and $1381 \mathrm{~cm}^{-1}$.
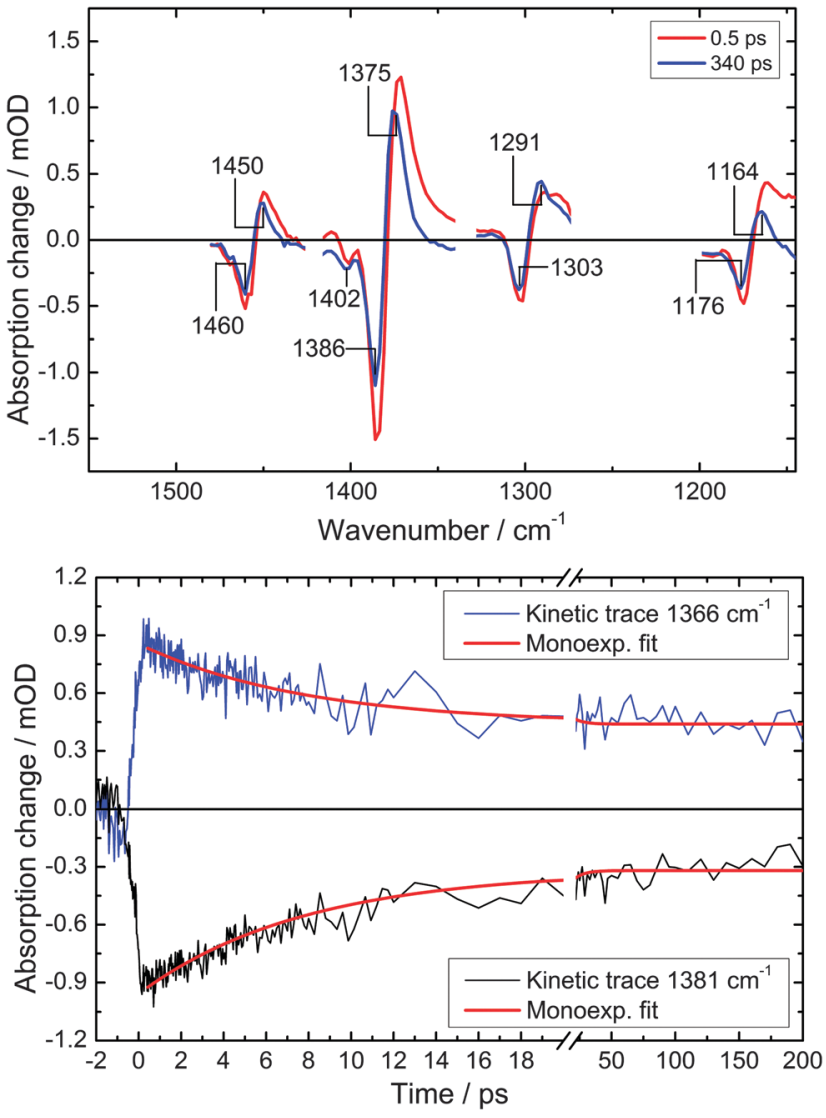

Fig. 4 Top: mid-IR difference absorption spectra at selected delay times ( 0.5 and $340 \mathrm{ps)}$ after photoexcitation at $330 \mathrm{~nm}$. Wavenumbers indicate the (apparent) peak positions at $340 \mathrm{ps}$. Bottom: kinetic trace at $1366 \mathrm{~cm}^{-1}$ (blue) and $1381 \mathrm{~cm}^{-1}$ (black) and global monoexponential fit with $\tau_{1}=7.8 \pm$ $0.4 \mathrm{ps}$ (red)

They show that after instantaneous absorption rise and bleach, respectively, the signal decreases within a few picoseconds and merges into stationary amplitude.

The stationary difference spectrum at 340 ps exhibits negative peaks at 1176, 1303, 1386, 1402 and $1460 \mathrm{~cm}^{-1}$ and positive peaks at $1164,1291,1375$ and $1450 \mathrm{~cm}^{-1}$. As in the TR-FTIR spectra the bleach peaks (neg.) are assigned to the electronic ground state and the positive vibrational bands are assigned to the long living excited state, the latter showing a rather uniform bathochromic shift of about $10 \mathrm{~cm}^{-1}$ with respect to the former. The difference spectrum at early times $(0.5 \mathrm{ps})$ is very similar to that at $340 \mathrm{ps}$, however, the respective difference bands are of larger amplitude with increased positive absorbance on the low-energy side. This feature is easily explained by vibrational cooling, i.e. the dissipation of the excess energy to the solvent: Anharmonic coupling between thermally populated low-energy vibrational modes and high-energy modes (fingerprint region) causes asymmetrically broadened vibrational bands. Thus, the observed apparent blueshift on the picosecond time scale indicates the cooling process of the vibrationally hot (unrelaxed) molecule in the excited electronic state. It should be noted that the spectral shapes (widths) of the positive bands in the transient spectra are not uniform since they are determined by band-specific parameters 


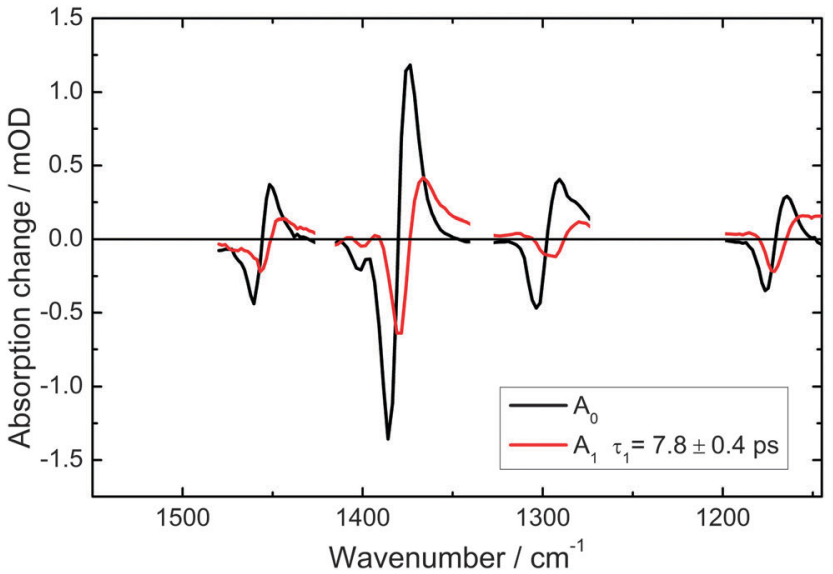

Fig. 5 Decay associated mid-IR spectra (DAS, according to eqn (2)), describing the cooling process $\left(A_{1}(\lambda)\right)$ and the "stationary" difference absorption spectrum $\left(A_{0}(\lambda)\right)$ (cf. text for details).

such as (1) spectral width (dephasing time) of the particular band in the excited electronic state and (2) the anharmonic coupling constants of the particular band to all other vibrational modes in the excited electronic state, especially to the low frequency modes.

Although the relation between vibrational temperature and time-dependency of the observed spectral changes is intrinsically non-exponential, the global fit according to eqn (2) is used in order to derive a characteristic time constant. This yields $\tau_{\mathrm{VR}}=7.8 \pm$ 0.4 ps with the decay associated spectrum $A_{1}(\lambda)$ given in Fig. 5 . It now describes the observed blue-shift of each product vibrational band (Fig. 4) in terms of monoexponentially decaying positive (low energy lobe) and negative (high energy lobe) absorbance strength as an approximation for the cooling process. $A_{0}(\lambda)$ is basically identical with the 340 ps difference spectrum, confirming the quality of the fit. Note the excellent match of $A_{0}(\lambda)$ and the microsecond-FTIR spectra (Fig. 3). No further, sub-ps time constant (as found in the transient UV/Vis data reported earlier $(380 \mathrm{fs})^{30}$ ) could be derived from the data. This might be due to the highly transitory character of the Franck-Condon state, leading to spectrally extremely broad infrared bands with possibly low absorption cross section. In addition the given (limited) time resolution and $\mathrm{S} / \mathrm{N}$-ratio add to this effect. Thus the IR data together with the transient UV/Vis data reported earlier (ref. 30) indicate that ultrafast intersystemcrossing (ISC), internal conversion (IC) and thermalization via intramolecular vibrational relaxation (IVR) lead to the vibrationally hot ${ }^{3} \mathrm{~A}$ excited state within less than $400 \mathrm{fs}$. Subsequently the excess energy of about $15500 \mathrm{~cm}^{-1}$ (according to $\lambda_{\mathrm{exc}}=330 \mathrm{~nm}$ and $\lambda_{\text {Fluor }}=675 \mathrm{~nm}^{30}$ ) is dissipated into the solvent.

The ultrafast dynamics, i.e. ISC within a few hundred femtoseconds followed by vibrational cooling on the picosecond time scale is typical for photoexcited transition-metal complexes ${ }^{36}$ and has been frequently reported. ${ }^{21,35}$ In addition, it turns out that the femtosecond transient IR spectra, by their strongly structured spectra, allow for a much clearer identification of the cooling/relaxation process as compared to broad and unstructured UV/Vis spectra, where corresponding spectral broadening and shifts are often much weaker or not even detectable as in our earlier work ${ }^{30}$ on this complex.

\section{Conclusions}

This paper reports on the first characterization of electronically excited states in a trinuclear Pd complex investigated by a combination of time-resolved IR spectroscopy and quantum chemical calculations. Step-scan FTIR and femtosecond IR spectroscopy are chosen to cover a time range from femtoseconds to microseconds. The same vibrational spectrum is obtained for the whole time range (from some ps to about $2 \mu \mathrm{s}$ ) indicating that within less than $400 \mathrm{fs}$ a long-lived electronic state is populated. From the TR-FTIR spectra the decay time of this long-lived excited state obtained via luminescence spectroscopy is confirmed. Quantum chemical calculations performed for the excited electronic states show that the lowest energy state is of ${ }^{3} \mathrm{~A}$ symmetry. Calculated IR spectra for the optimized ${ }^{3} \mathrm{~A}$ state match very well with the experimentally observed ones in contrast to the spectra calculated for the energetically higher ${ }^{3} \mathrm{~B}$ state. Thus an unambiguous assignment of the experimentally observed long-lived triplet state to $\mathrm{a}^{3} \mathrm{~A}$ state can be given in addition to the assignment via excited state electronic absorption. ${ }^{30}$

Furthermore the femtosecond transient IR data reveal vibrational cooling characterized by a monoexponential time constant of $7.8 \mathrm{ps}$ and no further changes up to $1 \mathrm{~ns}$. Thus the vibrational excess energy dissipates on a picosecond time scale.

The presented extensive spectroscopic and theoretical studies on a highly symmetric trinuclear Pd complex allow an assignment of electronically excited states and its dynamical behaviour. A longlived electronically excited state is formed by interaction of the three Pd atoms. The geometry of the complex changes from $D_{3}$ symmetry in the electronic ground state to $C_{2}$ symmetry in the ${ }^{3} \mathrm{~A}$ excited state, i.e. a pseudo-Jahn-Teller effect is observed. The trinuclear Pd complex has proved to be a rewarding object to study photophysics of a oligonuclear metal complex by the interplay of experiment and theory. The application of a variety of spectroscopic methods (UV/Vis, luminescence, molecular beam $)^{30}$ including now the structure sensitive IR techniques in combination with high level DFT and TDDFT calculations provide a detailed analysis on the structural changes by going from the electronic ground to an electronically excited state. These investigations are a starting point for further examples of interacting metal centers which can be of photophysical, magnetic or catalytical relevance.

\section{Acknowledgements}

Financial support by the German Science Foundation (DFG) and Collaborative Research Center CRC/Transregio 88, "Cooperative effects in homo- and heterometallic complexes (3MET)" is gratefully acknowledged (Projects B4, C1, C2, C4).

\section{Notes and references}

1 H. B. Gray and A. W. Maverick, Science, 1981, 214, 1201.

2 F. Y. Pétillon, P. Schollhammer, J. Talarmin and K. W. Muir, Coord. Chem. Rev., 1998, 178-180, 203-247.

3 D. C. Powers and T. Ritter, Acc. Chem. Res., 2012, 45, 840-850.

4 M. H. Pérez-Temprano, J. A. Casares and P. Espinet, Chem. - Eur. J., 2012, 18, 1864-1884. 
5 S. Serroni, S. Campagna, F. Puntoriero, F. Loiseau, V. Ricevuto, R. Passalacqua and M. Galletta, C. R. Chim., 2003, 6, 883-893.

6 J. A. Faiz, R. M. Williams, M. J. J. Pereira Silva, L. De Cola and Z. Pikramenou, J. Am. Chem. Soc., 2006, 128, 4520-4521.

7 T. Suzuki, T. Nakagawa, K. Ohkubo, S. Fukuzumi and Y. Matsuo, Chem. Sci., 2014, 5, 4888-4894.

8 S. Tschierlei, M. Presselt, C. Kuhnt, A. Yartsev, T. Pascher, V. Sundström, M. Karnahl, M. Schwalbe, B. Schäfer, S. Rau, M. Schmitt, B. Dietzek and J. Popp, Chem. - Eur. J., 2009, 15, 7678-7688.

9 B. Dietzek, W. Kiefer, J. Blumhoff, L. Böttcher, S. Rau, D. Walther, U. Uhlemann, M. Schmitt and J. Popp, Chem. - Eur. J., 2006, 12, 5105-5115.

10 I. Bratko and M. Gómez, Dalton Trans., 2013, 42, 10664-10681. 11 J. Park and S. Hong, Chem. Soc. Rev., 2012, 41, 6931-6943.

12 R. M. Haak, S. J. Wezenberg and A. W. Kleij, Chem. Commun., 2010, 46, 2713-2723.

13 O. R. Luca and R. H. Crabtree, Chem. Soc. Rev., 2013, 42, 1440-1459.

14 N. Huse, H. Cho, K. Hong, L. Jamula, F. M. F. de Groot, T. K. Kim, J. K. McCusker and R. W. Schoenlein, J. Phys. Chem. Lett., 2011, 2, 880-884.

15 S. E. Canton, K. S. Kjær, G. Vankó, T. B. van Driel, S.-I. Adachi, A. Bordage, C. Bressler, P. Chabera, M. Christensen, A. O. Dohn, A. Galler, W. Gawelda, D. Gosztola, K. Haldrup, T. Harlang, Y. Liu, K. B. Møller, Z. Németh, S. Nozawa, M. Pápai, T. Sato, T. Sato, K. Suarez-Alcantara, T. Togashi, K. Tono, J. Uhlig, D. A. Vithanage, K. Wärnmark, M. Yabashi, J. Zhang, V. Sundström and M. M. Nielsen, Nat. Commun., 2015, 6, DOI: $10.1038 /$ ncomms7359.

16 C. Bressler, C. Milne, V. T. Pham, A. ElNahhas, R. M. van der Veen, W. Gawelda, S. Johnson, P. Beaud, D. Grolimund, M. Kaiser, C. N. Borca, G. Ingold, R. Abela and M. Chergui, Science, 2009, 323, 489-492.

17 G. Vankó, A. Bordage, M. Pápai, K. Haldrup, P. Glatzel, A. M. March, G. Doumy, A. Britz, A. Galler, T. Assefa, D. Cabaret, A. Juhin, T. B. van Driel, K. S. Kjær, A. Dohn, K. B. Møller, H. T. Lemke, E. Gallo, M. Rovezzi, Z. Németh, E. Rozsályi, T. Rozgonyi, J. Uhlig, V. Sundström, M. M. Nielsen, L. Young, S. H. Southworth, C. Bressler and W. Gawelda, J. Phys. Chem. C, 2015, 119, 5888-5902.

18 S. E. Canton, X. Zhang, L. M. Lawson Daku, A. L. Smeigh, J. Zhang, Y. Liu, C.-J. Wallentin, K. Attenkofer, G. Jennings, C. A. Kurtz, D. Gosztola, K. Wärnmark, A. Hauser and V. Sundström, J. Phys. Chem. C, 2014, 118, 4536-4545.

19 A. L. Smeigh, M. Creelman, R. A. Mathies and J. K. McCusker, J. Am. Chem. Soc., 2008, 130, 14105-14107.

20 P. Tourón Touceda, S. Mosquera Vázquez, M. Lima, A. Lapini, P. Foggi, A. Dei and R. Righini, Phys. Chem. Chem. Phys., 2012, 14, 1038-1047.
21 M. M. N. Wolf, R. Gross, C. Schumann, J. A. Wolny, V. Schünemann, A. Døssing, H. Paulsen, J. J. McGarvey and R. Diller, Phys. Chem. Chem. Phys., 2008, 10, 4264-4273.

22 J. R. Schoonover, G. F. Strouse, K. M. Omberg and R. B. Dyer, Comments Inorg. Chem., 1996, 18, 165.

23 M. W. George and J. J. Turner, Coord. Chem. Rev., 1998, 177, 201.

24 J. M. Butler, M. W. George, J. R. Schoonover, D. M. Dattelbaum and T. J. Meyer, Coord. Chem. Rev., 2007, 251, 492-514.

25 B. D. Moore, M. Poliakoff, M. B. Simpson and J. J. Turner, J. Phys. Chem., 1985, 89, 850.

26 P. Chen, K. M. Omberg, D. A. Kavaliunas, J. A. Treadway, R. A. Palmer and T. J. Meyer, Inorg. Chem., 1997, 36, 954.

27 K. M. Omberg, J. R. Schoonover, J. A. Treadway, R. M. Leasure, R. B. Dyer and T. J. Meyer, J. Am. Chem. Soc., 1997, 119, 7013-7018.

28 A. Gabrielsson, F. Hartl, H. Zhang, J. R. L. Smith, M. Towrie, A. Vlček and R. Perutz, J. Am. Chem. Soc., 2006, 128, 4253.

29 F. Armbruster, J. Meyer, A. Baldes, P. Ona-Burgos, I. Fernández and F. Breher, Chem. Commun., 2011, 47, 221.

30 Y. Schmitt, K. Chevalier, F. Rupp, M. Becherer, A. Grün, A. M. Rijs, F. Walz, F. Breher, R. Diller, M. Gerhards and W. Klopper, Phys. Chem. Chem. Phys., 2014, 16, 8332-8338.

31 T. P. Dougherty and E. J. Heilweil, Chem. Phys. Lett., 1994, 227, 19-25.

32 P. Rudolf, F. Kanal, J. Knorr, C. Nagel, J. Niesel, T. Brixner, U. Schatzschneider and P. Nuernberger, J. Phys. Chem. Lett., 2013, 4, 596-602.

33 J. B. Asbury, K. Hang, J. S. Yeston, J. G. Cordaro, R. G. Bergman and T. Q. Lian, J. Am. Chem. Soc., 2000, 122, 12870-12871.

34 E. M. S. Maôas, R. Kananavicius, P. Myllyperkio, M. Pettersson and H. Kunttu, J. Am. Chem. Soc., 2007, 129, 8934-8935.

35 D. Imanbaew, Y. Nosenko, C. Kerner, K. Chevalier, F. Rupp, C. Riehn, W. R. Thiel and R. Diller, Chem. Phys., 2014, 442, 53-61.

36 A. Vlček, Coord. Chem. Rev., 2000, 200, 933-977.

37 P. S. Wagenknecht and P. C. Ford, Coord. Chem. Rev., 2011, 255, 591-616.

38 F. Peters, J. Herbst, J. Tittor, D. Oesterhelt and R. Diller, Chem. Phys., 2006, 323, 109-116.

39 TURBOMOLE V6.6 2014, a development of University of Karlsruhe and Forschungszentrum Karlsruhe $\mathrm{GmbH}$, 1989-2007, TURBOMOLE GmbH, 2007, available from http://www.turbomole.com.

40 C. van Wüllen, J. Comput. Chem., 2011, 32, 1195-1201.

41 P. Deglmann, F. Furche and R. Ahlrichs, Chem. Phys. Lett., 2002, 362, 511-518.

42 I. B. Bersuker, Chem. Rev., 2013, 113, 1351-1390.

43 M. K. Kesharwani, B. Brauer and J. M. L. Martin, J. Phys. Chem. A, 2015, 119, 1701-1714. 